\title{
Crystal Lattices Reconstruction from Moiré Aliased Scanning Transmission Electron Microscopy Electron Micrograph
}

\author{
Alexandre Pofelski ${ }^{1}$ and Gianluigi Botton ${ }^{2}$ \\ ${ }^{1}$ McMaster University, Canada, ${ }^{2}$ Department of Materials Science and Engineering, McMaster \\ University, Hamilton, ON, Canada, Canada
}

The effect sampling on the acquisition of a Scanning Transmission Electron Microscopy (STEM) electron micrograph is often neglected, since STEM is mostly applied on nanometer sized regions that are usually largely oversampled. The Whittaker-Nyquist-Kotel'nikov-Shannon sampling theorem $[1,2,3,4]$ is, in this case respected, and the sampling scheme does not require any special attention. Nevertheless, undersampling in STEM is surprisingly common when imaging crystalline samples. As the lattice parameter of crystals is usually in the range of Angstroms, using a pixel spacing of the same order of magnitude (or larger) for the scanning grid will inevitably lead to the undersampling of the resolved crystalline lattice. In such conditions, the undersampled crystal periodicities are irreversibly distorted, causing any large field-of-view STEM electron micrograph to be technically non usable on crystalline materials.

However, strategies exist for recovering the original features from undersampled STEM electron micrographs. For example, compressed sensing methods have been successfully applied to reconstruct the crystal lattices from its undersampled electron micrograph by randomizing the scanning process and using the incoherence of the sampler with the imaged features [5]. In this study, another strategy is proposed by intentionally generating aliasing artifacts and correcting them afterwards (hereafter referred to as Moiré sampling recovery [6]). The context of application of the Moiré sampling recovery is restricted to the socalled sparse bandwidth-limited periodic functions fitting the application of crystalline materials imaged in STEM. Building upon previous work $[7,8,9,10,11]$, the recovery of the crystalline lattices of a silicon sample from its undersampled STEM Moiré hologram is presented in Fig. 1. During the undersampling acquisition, the aliasing artifact transforms each crystalline wave vector (or reflection) to its corresponding Moiré wave vector (or reflection) through the sampling vector [12,13] forming the STEM Moiré hologram in Fig. 1 a). Knowing the sampling vectors for each resolved crystalline reflection, the aliasing shifts on all Moiré reflections from Fig. 1 b) can be reverted, as shown in Fig. $1 \mathrm{c}$ ), with the condition that each Moiré reflection is isolated. Applying the correction, the silicon crystal reflections are properly repositioned in Fourier space leading to the reconstruction of the silicon crystalline lattices in real space (see Fig 1.d)). A comparison between an experimental high resolution STEM electron micrograph and the numerically reconstructed micrograph is highlighted in Fig. 2. The silicon crystal structure is clearly recognizable, and the fine features, such as the silicon dumbbells along the direction, are also properly recovered. The great interest of the Moiré sampling recovery method is to extend the application of STEM on crystalline materials for low magnifications, thus avoiding the acquisition of multiple high resolution STEM electron micrographs at high magnification to cover the same field of view as the STEM Moiré hologram [13]. 

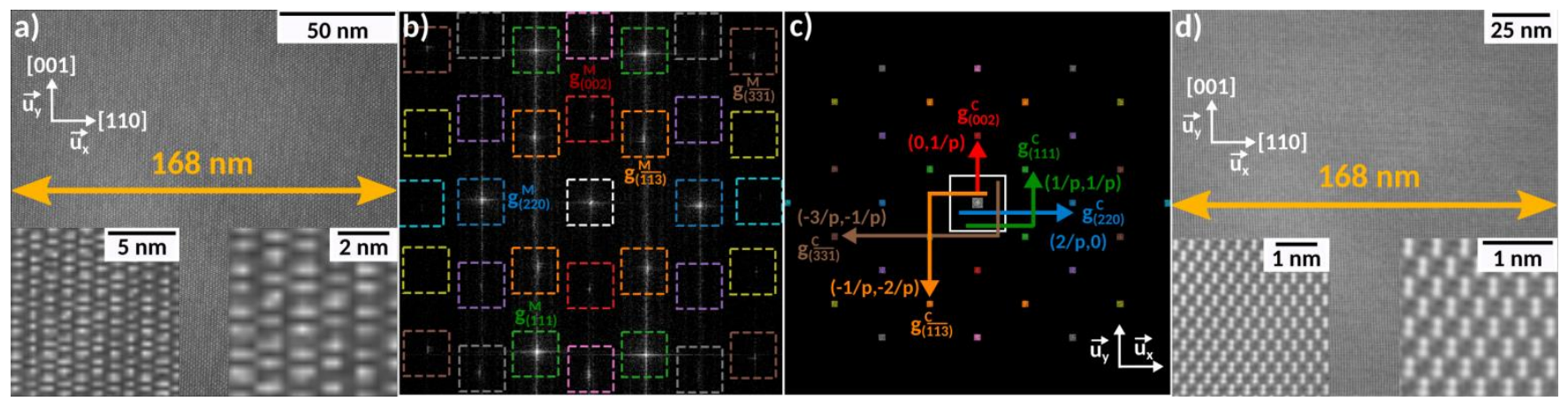

Figure 1. Application of the Moiré sampling recovery method. a) STEM Moiré hologram recorded on a silicon sample oriented along the [1-10] direction with 512 x 512 pixels and a pixel spacing of $329 \mathrm{pm}$. b) Fourier transform of the STEM Moiré hologram a) with the Moiré reflections gXM isolated from each other. The (111),(002),(220),(-1-1-3) and (-3-3-1) reflections are highlighted as examples. c) Correction of the aliasing artifact by applying the opposite of the sampling vector on each Moire reflection to form their respective crystalline reflections $\mathrm{gXC}$. The transformation on the five highlighted reflections from b) are displayed in their respective colour code. d) Inverse Fourier transform of c) revealing the reconstructed silicon crystal lattices in real space. The numerical reconstructed micrograph is composed of $7680 \times 7680$ pixels with a pixel spacing of $22 \mathrm{pm}$ and has the same field of view as the STEM Moiré hologram a).

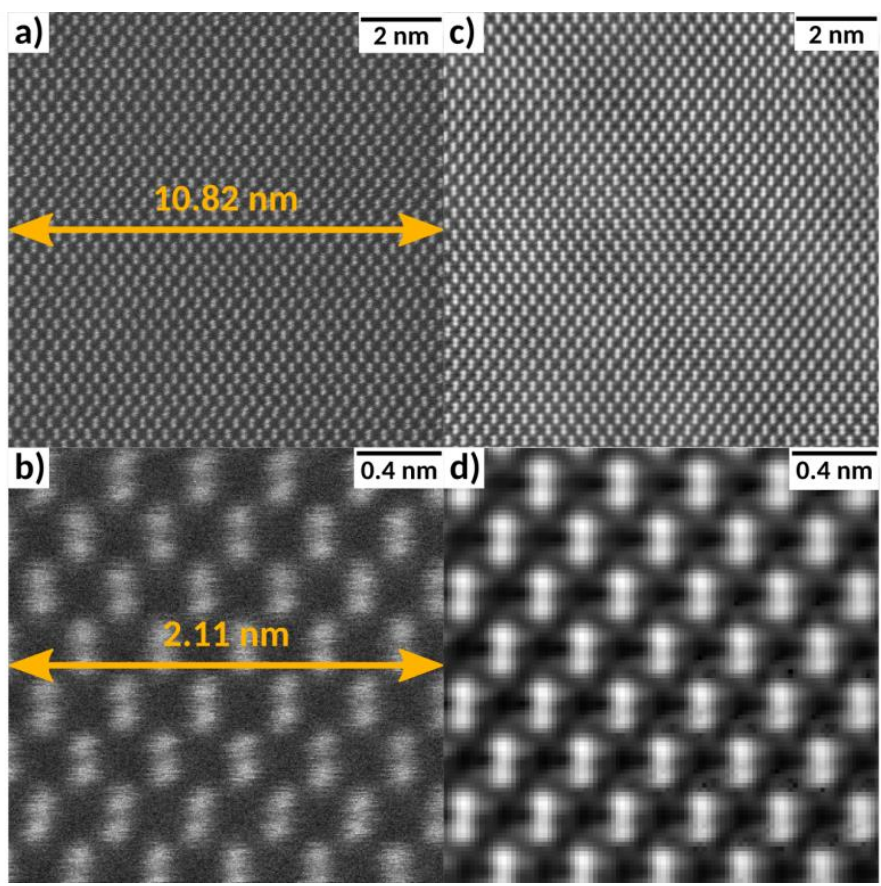

Figure 2. Comparison between a 2048 x 2048 pixels experimental high resolution STEM micrograph with a pixel spacing of $5.3 \mathrm{pm}$ (displayed in a) and b)) recorded on a silicon sample with the reconstruction from Fig. 1 (shown in c) and d)) cropped for the same field of view. 


\section{References}

[1] J.M. Whittaker, The Fourier Theory of the Cardinal Function, Proc. Edinburgh Math. Soc. 1 (1928) $169-176$.

[2] H. Nyquist, Certain Topics in Telegraph Transmission Theory, Trans. Am. Inst. Electr. Eng. 47 (1928) $617-644$.

[3] V.A. Kotel'nikov, O propusknoj sposobnosti “efira" i provoloki v elektrosvjazi, Izd. Red. Upr. Svyazi RKKA. (1933) 1-23.

[4] C.E. Shannon, Communication in the Presence of Noise, Proc. IRE. 37 (1949) 10-21.

[5] A. Béché, B. Goris, B. Freitag, J. Verbeeck, Development of a fast electromagnetic beam blanker for compressed sensing in scanning transmission electron microscopy, Appl. Phys. Lett. 108 (2016) 093103. [6] A. Pofelski, G.A. Botton, to be submitted (Ultramicroscopy)

[7] C. Rader, Recovery of undersampled periodic waveforms, IEEE Trans. Acoust. 25 (1977) 242-249.

[8] A.J. Silva, Reconstruction of Undersampled Periodic Signals, Massachusetts Institute of Technology, 1986.

[9] D.M. Simpson, Reconstruction of undersampled signals and alignment in the frequency domain, Imperial College London, 1988.

[10] P.J.B. Koeck, Aliasing Correction of Undersampled Crystal Images, in: 12th Eur. Congr. Electron Microsc., 2000: pp. 395-396.

[11] V. Grillo, F. Rossi, STEM_CELL: A software tool for electron microscopy. Part 2 analysis of crystalline materials, Ultramicroscopy. 125 (2013) 112-129.

[12] A. Pofelski, S.Y. Woo, B.H. Le, X. Liu, S. Zhao, Z. Mi, S. Löffler, G.A. Botton, 2D strain mapping using scanning transmission electron microscopy Moiré interferometry and geometrical phase analysis, Ultramicroscopy. 187 (2018) 1-12.

[13] A. Pofelski, S. Ghanad-Tavakoli, D.A. Thompson, G.A. Botton, Sampling optimization of Moiré geometrical phase analysis for strain characterization in scanning transmission electron microscopy, Ultramicroscopy. 209 (2020) 112858.

[13] The authors are grateful to the Natural Sciences and Engineering Research Council for a Discovery Grant supporting this work. 